\title{
Research on Application of Security Technology in Network Engineering Deng Tian
}

\author{
NanChang Institute of Science \& Technology
}

\section{Keywords: Security technology; Network engineering; Encryption application}

\begin{abstract}
With the rapid development of computer network, the sharing of various network resources is further strengthened, and the information security problem is becoming more and more prominent. In fact, resource sharing and security has always been a contradiction. In an open network environment, a large number of information sharing is bound to provide criminals with the target of attack. As far as the current situation of network security is concerned, the main security threats of computer network system come from three aspects: hacker attack, computer virus and denial of service attack. Hacker attacks emerged as early as the host terminal era, As the network continues to evolve, modern hackers have changed from system-based attacks to network-based attacks. Based on the study of the security framework of the encryption system in the network system, this paper puts forward the programming method of network security application based on the encryption machine framework. The proposed encryption method proposed in the paper can be applied to the current network security encryption architecture; can provide easy-to-use and high-performance network security solutions for service.
\end{abstract}

\section{Introduction}

In today's network era, network security is very important for our work and daily life, with the development of the times and the continuous progress of society, we believe that the requirements for network security will be higher and higher in society, while the network security technology is the most effective ways and means to provide security for the network. This paper illustrated the definition content of network security technology, and as a theoretical basis, combined with the relevant work experience of network security technology from authors, to analyzes and explores the specific application of security technology in network engineering. We hope to help the rational application of the network security technology in the network engineering, to promote the healthy development of network security. Network is an ecological system composed of human and network information. In this study, we use the theory of ecology to analyze the essence of network ecosystem by using the method of analogy from the natural ecosystem, and to study the law of network ecosystem. We seek the most fundamental method of solving the crisis of network ecosystem and maintain the ecological balance of the network to create a harmonious and balanced ecological environment for network and ensure the healthy and sustainable development of network ecosystems.

\section{The Definition of Network Security Technology}

The so-called network security technology can be defined from two aspects. On the one hand, from the physical point of view, carrying out security protection from network equipment and network accessories. In order to prevent the network equipment and network-related configuration from the fire and radiation effects, we need to develop related security management system of network equipment to ensure that the power of network equipment and related accessories can not be interrupted. At the same time, it is necessary to develop strict control and management for the user's access, we can set some fingerprint recognition and other security measures to ensure that the network will not be free to access. On the other hand, from the abstract point of view, that is the prevention and processing of computer virus. In order to ensure that the network is not affected by the invasion and damage of computer viruses, we should set up a safe and effective prevention software for computer network virus. In addition, we need to make a rigorous inspection and 
control in regard to the information received and issued in the use of the network, and pay attention to the backup processing of information to ensure that the the minimum losses suffered by the virus. In recent years, the technologies related to the network security emerge in endlessly, such as virus firewall technology and secret security management and intelligent access control technology, smart card technology, digital signature technology, intelligent authentication technology and some other technical means. These new technologies can effectively protect the network security, and provide multiple securities for the overall security of network engineering, Effectively preventing the virus intrusion or impersonate user access from affecting the safety of network engineering.

\section{Analysis on Application of Security Technology in Network Engineering}

Analysis on the Requirements of Security Technology from Users. Generally speaking, end users of a network engineering are commonly more than one hundred, this type of terminal users have a very high demand for network security technology protection in the use of the network. Such as the use of some files, the sending and use of software, the use of office equipment connected to the network, the use and management of computer user accounts, the use authentication of user, network access, data transmission, hardware management and the management of system and network. When Users are doing these processes and operations, focusing on the security and stability of network greatly. At the same time, because the number of end users is generally large, so the network security technology products will also pay special attention to the number of users in the aspects of technology research and capital injection.

Analysis on the Requirement of Security Technology due to Network Development. From the point of view of the actual effect of network security technology, in order to make the effect of network expansion more efficient and stable, at present, the most advanced model used is one kind of expansion mode called star-type network structure. That is, the host is treated as the core area of the entire network project, using some of the central connection system and the switch system to establish the connection with host network engineering. This model of connection can be more efficiently and reasonably to provide network services for end-users, but also to reconfigure network resources reasonably. On the other hand, this kind of star connection model can also effectively reduce the damage of failures to the entire network engineering.

The Isolation of Network. The so-called network isolation, in fact, is the virus firewall technology coming for network security, today this technology also is the most widely used network security technology measures. Firewall technology can effectively prevent some network information not got permission or strictly prohibited into the protected internal network project. At the same time, based on the specific characteristics of the network system, we can make appropriate change for the design of firewall technology to adapt to itself, such as shielding the router or host and dual-host structure shielding and so on.

\section{Application Status of Encryption}

Host encryption machine is a domestic self-developed host encryption device getting through the identification and approval of national commercial password administration, encryption machine and host use TCP / IP protocol communication, so encryption machine have no any special requirements on the operation system of host machine. Through the application of encryption machines, it provides computer network systems with secure data communications services, to prevent all kinds of fraud conduct from happening online .

Generally speaking, there are four main functions of the encryption module: Hardware encryption components, key management menu, encryption background process, Encryption monitoring procedures and background monitoring process.

The main function of hardware encryption components is to achieve all kinds of cryptographic algorithms, security keys, such as the root key of CA and so on. By providing the front-end API for the application system to build the cryptographic development connector, the application system encrypts the encryption service by using the cryptographic foreground API , while the encryption 
front-end API is provided in the form of a standard C library. The encryption machine supports a variety of commonly used international cipher algorithms, currently, the widely used symmetric encryption algorithm are DES and 3DES.

For the system applications of the need to use encryption system for network information encryption, generally involving in financial transactions or sensitive information, such as the telecommunications charge system, the bank trading system, government information release system and so on. In these systems, if you use non-encrypted system network security settings, will inevitably have a risk. If you use a simple software encryption settings, the probability of system security being cracked will be great. Therefore, this paper mainly illustrates how to use hardware-level encryption technology for these networks in need of encryption machine to achieve the purpose of preventing security risks.

Generally speaking, the encryption machine uses a three-tier key system, and functions of the different keys (especially the work of the key) has been done a strict restrictions on the use. The general key stratification system is shown in Fig. 1 below.

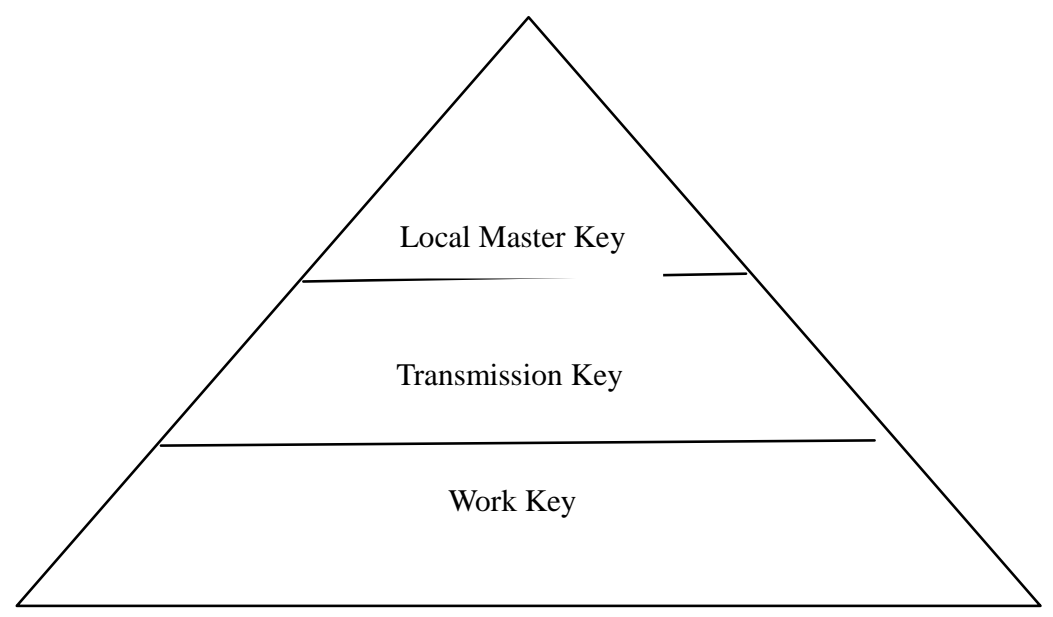

Figure 1. Key stratification system

\section{Conclusion}

The considerations of network engineering in security should pay attention to network data and virus problems simultaneously, as well as fire and radiation and other issues. Network security technology in order to achieve the safety of the user network, access resources, the protection system, the prevention of virus intrusion and filtering information have been effectively protected. However, network security technology, after all, is only one aspect of network engineering security, when using network security technology, we should artificially strengthen the awareness of protecting network engineering, setting relevant protection regulation and management measures to achieve the greatest degree to avoid the occurrence of network security risks. This paper, through the core-level master key, transmission master key and data exchange level work key, illustrating the application of 3 layer key system in the security of network. How to set the encryption machine in the application of network security and The principle of how to use the encryption machine to provide the foreground API for network security application is explained.

Project funding. Nanchang Intelligent Building Network Engineering Key Laboratory \& Specialty of Jiangxi Province (NGTSZY201001)

\section{References}

[1] Jia X, Wang C. The Security Routing Research for WSN in the Application of Intelligent Transport System. IEEE International Conference on Mechatronics and Automation. IEEE, 2006:2318-2323. 
[2] Jing Z J Z, Xinguang X G L. The Application Research of Information Hiding Technology in Network Security. 2009:208-212.

[3] Li B, Lv S J, Zhang Y S, et al. The application research of Cookies in network security. International Conference on Sensor Network Security Technology and Privacy Communication System. IEEE, 2013:152-155.

[4] $\mathrm{Xu} \mathrm{X,} \mathrm{Lu} \mathrm{J} \mathrm{X.} \mathrm{Application} \mathrm{of} \mathrm{WLAN} \mathrm{Information} \mathrm{Transmission} \mathrm{and} \mathrm{System} \mathrm{Security}$ Technology in the Smart Substation Network. Advanced Materials Research, 2013, 684:551-554.

[5] Jia L. The Research and Application of Network Security Technology in the University Network. Advanced Materials Research, 2013, 756-759(756-759):1686-1689.

[6] Li J, Wang H, Yu J. Research on the Application of CRFs Based on Feature Sets in Network Intrusion Detection. International Conference on Security Technology. IEEE Xplore, 2008:194-197.

[7] Lou Y F, Yuan Z J. The Research and Application of Key Technology on Wireless Multi-Hop Mesh Network. 2013, 791-793:1660-1663.

[8] Zhang P, Jiao X, Zhou R. Network Security Technology in P2P[J]. Advanced Research on Computer Science \& Information Engineering, 2011, 153:145-149.

[9] Li J. The Research and Application of Multi-Firewall Technology in Enterprise Network Security $[\mathrm{J}]$. International Journal of Software Engineering \& Its Applications, 2015, 9(5):153-162.

[10]Gong C. Research on the Application of Network Technology in the Dissemination and Creation of Literature. Applied Mechanics \& Materials, 2014, 687-691:1836-1839.

[11] Yi-Feng H U, Polytechnic S. Research on the Application of Network Security Technology in Campus Network. Earth Surface Processes \& Landforms, 1995, 39(6):88-91.

[12]Huang Y W, Tsai C H, Lin T P, et al. A testing framework for Web application security assessment. Computer Networks the International Journal of Computer \& Telecommunications Networking, 2005, 48(5):739-761. 